PROCEEDINGS OF THE

AMERICAN MATHEMATICAL SOCIETY

Volume 131, Number 3, Pages 835-843

S 0002-9939(02)06642-X

Article electronically published on September 17, 2002

\title{
INFINITELY MANY NONRADIAL SOLUTIONS TO A SUPERLINEAR DIRICHLET PROBLEM
}

\author{
HUGO ADUÉN AND ALFONSO CASTRO
}

(Communicated by David S. Tartakoff)

\begin{abstract}
In this article we provide sufficient conditions for a superlinear Dirichlet problem to have infinitely many nonradial solutions. Our hypotheses do not require the nonlinearity to be an odd function. For the sake of simplicity in the calculations we carry out details of proofs in a ball. However, the proofs go through for any annulus.
\end{abstract}

\section{INTRODUCTION}

Here we consider the Dirichlet problem

$$
\left\{\begin{aligned}
\Delta u+f(u)=0 & \text { in } \Omega, \\
u=0 & \text { on } \partial \Omega,
\end{aligned}\right.
$$

where $\Delta$ is the Laplacian operator, $\Omega$ is the unit ball in $\mathbf{R}^{n}, n \geq 2$, and $f: \mathbf{R} \rightarrow \mathbf{R}$ is a function of class $C^{1}$. We assume that $f$ is superlinear, i.e.,

$$
\lim _{|t| \rightarrow+\infty} \frac{f(t)}{t}=+\infty
$$

We also assume that there exist constants $\mu_{1}>0, \mu_{2}>0, \rho>0, \omega>1$, and $p \in(1,(n+2) /(n-2))$ such that for $|t| \geq \rho$,

$$
\mu_{1}\left|f^{\prime}(t)\right|^{\frac{p+1}{p-1}} \leq \Phi_{\omega}(t) \leq \Phi_{1}(t) \leq \mu_{2}\left(\frac{1}{2} t f(t)-F(t)\right) .
$$

Here $F(t):=\int_{0}^{t} f(s) d s$, and $\Phi_{s}(t):=2 n F(t)-s(n-2) t f(t)$, for all $t \in \mathbf{R}$. Finally, we assume that there exists $\mu_{3}>0$ such that

$$
t^{2} f^{\prime}(t)-t f(t) \geq \mu_{3} \quad \text { for } \quad|t| \geq \rho .
$$

The goal of this paper is to establish sufficient conditions for (1.1) to have infinitely many nonradial solutions (see Theorem 1 and Theorem 3 below). The existence of infinitely many radial solutions for (1.1) has been established by several authors assuming conditions related to (1.2)-(1.4) (see [2], [7] and [11]). The methods used in [7] or [11], however, shed no light on how to extend them to finding nonradial solutions. Actually, despite the intense development on radial solutions to problems like (1.1), results on the existence of nonradial solutions is nowhere

Received by the editors March 8, 2001 and, in revised form, October 15, 2001.

2000 Mathematics Subject Classification. Primary 35J20; Secondary 34B15.

Key words and phrases. Critical point, Morse index, nonradial solutions, Cwikel inequality, nonlinear elliptic equation. 
near as abundant. The reader is referred to [3], 4, [5], [6], and [10] for results on the existence of nonradial solutions to (1.1).

In order to state our main results we recall that the solutions to the equation (1.1) are the critical points of the functional $J: H_{0}^{1}(\Omega) \rightarrow \mathbf{R}$ defined by

$$
J(u)=\int_{\Omega}\left(\frac{1}{2}\|\nabla u\|^{2}-F(u)\right) d x
$$

where $H_{0}^{1}(\Omega)$ is the Sobolev space of square integrable functions in $\Omega$ having first order partial derivatives in $L^{2}(\Omega)$ and vanishing on $\partial \Omega$. Because of (1.3) the function $f$ has subcritical growth, i.e., there exist $A>0$ and $p \in(1,(n+2) /(n-2))$ such that

$$
|f(t)| \leq A\left(|t|^{p}+1\right) \quad \text { for all } \quad t \in \mathbf{R} .
$$

Hence the functional $J$ is of class $C^{1}$. Replacing (1.6) in (1.3) we see that there exists $B>0$ such that $\left|f^{\prime}(t)\right| \leq B\left(|t|^{p-1}+1\right)$, for all $t \in \mathbf{R}$. Therefore, $J$ is of class $C^{2}$. The gradient and Hessian of $J$ are given by

$$
\begin{aligned}
\langle\nabla J(u), v\rangle & =\int_{\Omega}(\nabla u \cdot \nabla v-f(u) v) d x, \\
\left\langle D^{2} J(u) v, w\right\rangle & =\int_{\Omega}\left(\nabla v \cdot \nabla w-f^{\prime}(u) v w\right) d x,
\end{aligned}
$$

respectively.

Our main result is the following.

Theorem 1. Let $\gamma:=2(p+1) /(n(p-1))$. Suppose there exist $M_{1}>0, \alpha \in(0, \gamma]$, and a sequence $\left\{u_{i}\right\}$ of solutions to (1.1) such that $J\left(u_{i}\right) \rightarrow+\infty$ as $i \rightarrow \infty$ and

$$
J\left(u_{i}\right) \leq M_{1} i^{\alpha} .
$$

If there exist $M_{2}>0, k_{1}>0$, and

$$
\delta \in\left(0, \frac{(n-1)(n+2-p(n-2))}{2(p+1)}\right)
$$

such that, for each positive integer $k \geq k_{1}$, the equation (1.1) has at most $M_{2} k^{\delta}$ radial solutions with $k$ interior nodal hypersurfaces, then (1.1) has infinitely many nonradial solutions.

In order to prove Theorem 1 we show that given $\epsilon>0$, adequately small, if $u$ is a radial solution to (1.1) with $k$ interior nodal hypersurfaces, then $J(u) \geq k^{\gamma+\epsilon}$, for $k$ sufficiently large (see Lemma 2.4 below). The proof relies heavily on the so-called Cwikel inequality (see 8]), which we state for the sake of completeness.

Theorem 2. There exists $C>0$ such that if $V \in L^{n / 2}(\Omega)$, then the number of negative eigenvalues of the operator $-\Delta+V$ on $H_{0}^{1}(\Omega)$, call it $\sigma(V)$, satisfies $\sigma(V) \leq C \int_{\Omega}\left|V_{-}\right|^{n / 2} d x$, where $V_{-}$is the negative part of $V$.

Our arguments do not need the nonlinearity $f$ to be an odd function. In fact, our next result exemplifies the applicability of Theorem 1 for $f$ not odd. If $f$ is odd, say $f(u)=|u|^{p-1} u$, one can prove the existence of infinitely nonradial solutions by reflecting positive solutions to (1.1) in regions that tile the ball (see 9]).

Theorem 3. Let $a \in \mathbf{R}$ and $1<p<\frac{n+2}{n}$. If $f(u)=|u+a|^{p-1}(u+a)$, then (1.1) has infinitely many nonradial solutions.

The proof of Theorem 3 is deferred to Section 4 . 


\section{Preliminary lemmas}

Throughout this section we use the fact that if $u$ is a radial solution of (1.1), then there exists $v \in C^{2}[0,1]$ such that $u(x)=v(\|x\|)$ and

$$
\left\{\begin{aligned}
v^{\prime \prime}(r)+\frac{n-1}{r} v^{\prime}(r)+f(v(r)) & =0, \quad r \in(0,1], \\
v^{\prime}(0)=v(1) & =0 .
\end{aligned}\right.
$$

Let

$$
E(r):=\frac{1}{2}\left|v^{\prime}(r)\right|^{2}+F(v(r)) .
$$

Differentiating the energy function $E$, and applying (2.1) one obtains

$$
E^{\prime}(r)=-\frac{n-1}{r}\left|v^{\prime}(r)\right|^{2} \leq 0 .
$$

Lemma 2.1. There exist positive constants $C_{1}, \bar{C}_{1}$ such that if $u$ is a radial solution of (1.1), then

$$
J(u) \geq C_{1}\left|v^{\prime}(1)\right|^{2}-\bar{C}_{1} .
$$

Proof. Since $u$ is a critical point of $J$, it follows that

$$
J(u)=\int_{\Omega}\left(\frac{1}{2} u(x) f(u(x))-F(u(x))\right) d x,
$$

which together with the definition of $v$ imply

$$
J(u)=n|\Omega| \int_{0}^{1} r^{n-1}\left(\frac{1}{2} v(r) f(v(r))-F(v(r))\right) d r .
$$

Now, multiplying (2.1) by $\left(r v^{\prime}+\frac{n-2}{2} v\right) r^{n-1}$ and integrating by parts over $[0,1]$ we obtain

$$
\left|v^{\prime}(1)\right|^{2}=\int_{0}^{1} r^{n-1} \Phi_{1}(v(r)) d r
$$

Thus, (2.4) follows from the last inequality in (1.3), (2.5) and (2.6).

Lemma 2.2. There exist positive constants $C_{2}$ and $d_{0}$ such that if $v$ is a solution to the second order differential equation in (2.1) with $|v(0)| \geq d_{0}$ and $v^{\prime}(0)=0$, then

$$
r_{i+1}-r_{i} \geq C_{2}\left|v^{\prime}\left(r_{i}\right)\right|^{\frac{1-p}{p+1}},
$$

where $0<r_{i}<r_{1+1} \leq 1$ are two consecutive zeros of $v$.

Proof. By hypotheses (1.2) and (1.3), there exist constants $c>0$ and $\rho_{1} \geq \rho$ such that for $|t| \geq \rho_{1}$ we have

$$
t f(t)>0 \quad \text { and } \quad|f(t)| \leq c t[F(t)]^{\frac{p-1}{p+1}} .
$$

Since $F(t) \rightarrow+\infty$ as $|t| \rightarrow+\infty$, there exists $\rho_{2} \geq \rho_{1}$ such that if $F(t) \geq \rho_{2}$, then $|t| \geq 2 \rho_{1}$. By the first inequality in (1.3) and Lemma 2.2 of [7, we see that

$$
E(t) \rightarrow+\infty \quad \text { and } \quad t^{n} E(t)+\frac{n-2}{2} t^{n-1} v(t) v^{\prime}(t) \rightarrow+\infty,
$$


uniformly for $t \in[\sqrt{2 n v(0) / f(v(0))}, 1]$ as $|v(0)|$ tends to $+\infty$. Hence there exists $d_{0}>0$ such that if $v$ is a solution to the second order differential equation in (2.1), with $v^{\prime}(0)=0$, and $|u(0)|>d_{0}$, then $2 E(1)=\left|v^{\prime}(1)\right|^{2} \geq 2 \rho_{2}$.

Let $t_{i} \in\left(r_{i}, r_{i+1}\right)$ be such that

$$
\left|v\left(t_{i}\right)\right|=\max \left\{|v(s)|: s \in\left(r_{i}, r_{i+1}\right)\right\} .
$$

Without loss of generality we may assume that $v\left(t_{i}\right)>0$. Since $\left|v^{\prime}(1)\right|^{2} \geq 2 \rho_{2}$, by (2.3), $F\left(v\left(t_{i}\right)\right) \geq \rho_{2}$. Hence $v\left(t_{i}\right) \geq 2 \rho_{1}$. Let $y_{i} \in\left(t_{i}, r_{i+1}\right)$ be such that $2 v\left(y_{i}\right)=v\left(t_{i}\right)$. From (2.1) and (2.8), for $t \in\left[t_{i}, y_{i}\right]$ we have

$$
-t^{n-1} v^{\prime}(t)=\int_{t_{i}}^{t} r^{n-1} f(v(r)) d r \leq c \int_{t_{i}}^{t} r^{n-1} v(r)[F(v(r))]^{\frac{p-1}{p+1}} d r .
$$

Since $F(v(r)) \leq\left[v^{\prime}\left(r_{i}\right)\right]^{2}$ for all $r \in\left[t_{i}, y_{i}\right]$ (see (2.3) $)$, we see that

$$
-v^{\prime}(t) \leq c\left(t-t_{i}\right)\left|v\left(t_{i}\right)\right|\left|v^{\prime}\left(r_{i}\right)\right|^{\frac{2(p-1)}{p+1}}, \quad \text { for } t \in\left[t_{i}, y_{i}\right] .
$$

Integrating on $\left[t_{i}, y_{i}\right]$, using that $2 v\left(y_{i}\right)=v\left(t_{i}\right)$, and taking $C_{2}=\sqrt{1 / c}$, the lemma follows.

Lemma 2.3. Let $d_{0}$ be as in Lemma 2.2. There exist positive constants $C_{3}, \bar{C}_{3}$, and $\hat{d} \geq d_{0}$ such that if $u$ is a radial solution to (1.1) with $|u(0)| \geq \hat{d}$ and having $j$ nodal interior hypersurfaces, then

$$
C_{3} j \leq \int_{\Omega}\left|f^{\prime}(u(x))\right|^{\frac{n}{2}} d x \leq \bar{C}_{3}\left(\left|v^{\prime}(1)\right|^{\frac{2}{\gamma}}+1\right) .
$$

Here, and in what follows, $\gamma$ is as defined in Theorem 1 .

Proof. Our proof of the first inequality in (2.11) is based on the fact that if $r_{i}<r_{i+1}$ are two consecutive zeros of $v$, then

$$
\int_{r_{i}}^{r_{i+1}} r^{n-1} \phi(r) d r<0
$$

where $\phi(r)=v(r) f(v(r))-f^{\prime}(v(r)) v^{2}(r)$. In order to prove (2.12) we let $t_{i}$ be as in (2.10), $a \in\left(r_{i}, t_{i}\right)$ be such that $v(a)=\rho_{1}$, and $b \in\left(t_{i}, r_{i+1}\right)$ be such that $v(b)=\rho_{1}$. By (2.9) and the fact that $F$ is bounded on $\left[0, \rho_{1}\right]$ we may assume that $-((n-1) / r) v^{\prime}(r)-f(v(r))<0$ for all $r \in\left[r_{i}, t_{i}\right]$. Hence $v$ is concave on $r \in\left[r_{i}, t_{i}\right]$. Thus $v(r) \geq v\left(t_{i}\right)\left(r-r_{i}\right) /\left(t_{i}-r_{i}\right)$. In particular $a-r_{i} \leq \rho_{1}\left(t_{i}-r_{i}\right) / v\left(t_{i}\right)$. Let $L_{1}=\max \left\{|\phi(r)| ;|r| \leq \rho_{1}\right\}$. Hence

$$
\begin{aligned}
\int_{r_{i}}^{t_{i}} r^{n-1} \phi(r) d r & \leq \int_{r_{i}}^{a} r^{n-1} L_{1} d r+\int_{a}^{t_{i}} r^{n-1}\left(-\mu_{3}\right) d r \\
& \leq a^{n-1}\left[\int_{r_{i}}^{a}\left(L_{1}+\mu_{3}\right) d r-\mu_{3} \int_{r_{i}}^{t_{i}} d r\right] \\
& \leq a^{n-1}\left(t_{i}-r_{i}\right)\left[\frac{\left(L_{1}+\mu_{3}\right) \rho_{1}}{v\left(t_{i}\right)}-\mu_{3}\right]<0,
\end{aligned}
$$

where we have used that for $|u(0)|$ sufficiently large $v\left(t_{i}\right)>\left(1+\left(L_{1} / \mu_{3}\right)\right) \rho_{1}$ (see (2.9)). The constant $\mu_{3}$ is given by the hypothesis (1.4).

Now let

$$
s=2+\frac{\mu_{3}}{2 L_{1}}
$$


Let $L_{2}=\max \left\{|F(t)| ; 0 \leq t \leq(3 / 2)^{n-1} s \rho_{1}\right\}$ and let $c \in\left[t_{i}, b\right]$ be such that $v(c)=$ $s \rho_{1}$. Since $|F(v(t))| \leq L_{2}$ for $r \in\left[c, r_{i+1}\right]$, by (2.9) we see that for large values of $|u(0)|$ we have

$$
\left(v^{\prime}(r)\right)^{2} \geq\left(v^{\prime}\left(r_{i+1}\right)\right)^{2}-L_{2} \geq\left(v^{\prime}\left(r_{i+1}\right)\right)^{2} / 2 .
$$

Integrating $v^{\prime}$ on $\left[b, r_{i+1}\right]$ we have

$$
r_{i+1}-b \leq\left(2 \rho_{1}\right) /\left|v^{\prime}\left(r_{i+1}\right)\right| .
$$

For $|u(0)|$ large enough, from (2.9), we may assume that $r_{i+1} v^{\prime}\left(r_{i+1}\right) \geq 4 s \rho_{1}$. Integrating (2.1) on $\left[r, r_{i+1}\right]$ we see that

$$
\begin{aligned}
-r^{n-1} v^{\prime}(r) & =-r_{i+1}^{n-1} v^{\prime}\left(r_{i+1}\right)-\int_{r}^{r_{i+1}} s^{n-1} f(v(s)) d s \\
& \leq-r_{i+1}^{n-1} v^{\prime}\left(r_{i+1}\right)-r_{i+1}^{n-1}\left(r_{i+1}-r\right) K \\
& \leq-2 r_{i+1}^{n-1} v^{\prime}\left(r_{i+1}\right),
\end{aligned}
$$

where $K=\min \{f(u) ; u \geq 0\}$. Hence for $r \in\left[\hat{r}, r_{i+1}\right]$, with $\hat{r} \equiv r_{i+1}-\left(s \rho_{1}\right) /\left|v^{\prime}\left(r_{i+1}\right)\right|$, we have

$$
-v^{\prime}(r) \leq\left|v^{\prime}\left(r_{i+1}\right)\right|\left(r_{i+1} / r\right)^{n-1} \leq\left|v^{\prime}\left(r_{i+1}\right)\right|(4 / 3)^{n-1} .
$$

Integration of $v^{\prime}$ on $\left[\hat{r}, r_{i+1}\right]$ yields $v(\hat{r}) \leq(4 / 3)^{n-1} s \rho \leq(3 / 2)^{n-1} s \rho$. Arguing as in (2.13), by (2.14), we conclude that

$$
\begin{gathered}
\int_{t_{i}}^{r_{i+1}} r^{n-1} \phi(r) d r \leq-\int_{\hat{r}}^{b} r^{n-1} \mu_{3} d r+\int_{b}^{r_{i+1}} r^{n-1} L_{1} d r \\
\leq \frac{\rho_{1}}{\left|v^{\prime}\left(r_{i+1}\right)\right|}\left[-\mu_{3} \hat{r}^{n-1}(s-2)+2 L_{1} r_{i+1}^{n-1}\right]<0 .
\end{gathered}
$$

Thus, from (2.13) and (2.18), we have (2.12).

For $i=1,2, \ldots, j+1$, we define $A_{i}=\left\{x \in \Omega ; r_{i} \leq\|x\| \leq r_{i+1}\right\}$, and $u_{i}=u \chi_{i}$, where $\chi_{i}$ is the characteristic function of $A_{i}$. By (2.12) and (1.7) we see that

$$
\begin{aligned}
\left\langle D^{2} J(u) u_{i}, u_{i}\right\rangle & =\int_{\Omega}\left(\left\|\nabla u_{i}\right\|^{2}-f^{\prime}(u(x)) u_{i}^{2}(x)\right) d x \\
& =\int_{\Omega}\left(u_{i}(x) f\left(u_{i}(x)\right)-f^{\prime}(u(x)) u_{i}^{2}(x)\right) d x \\
& =\left|S_{n}\right| \int_{r_{i}}^{r_{i+1}} r^{n-1} \phi(r) d r<0,
\end{aligned}
$$

where $S_{n}$ denotes the measure of the unit sphere in $\mathbf{R}^{n}$. Thus $u$ has Morse index greater than or equal to $j+1$. Hence by Cwikel's inequality we have $j \leq C \int_{\Omega}\left|f^{\prime}(u(x))\right|^{\frac{n}{2}} d x$, which proves the first inequality in (2.11). On the other hand, Hölder's inequality and (1.3) yield

$$
\begin{aligned}
\int_{\Omega}\left|f^{\prime}(u(x))\right|^{\frac{n}{2}} d x & \leq K_{1} \int_{\Omega}\left[\Phi_{1}(u(x))+1\right]^{\frac{1}{\gamma}} d x \\
& \leq K_{2}\left(\int_{\Omega}\left[\Phi_{1}(u(x))+1\right] d x\right)^{\frac{1}{\gamma}} \\
& \leq K_{3}\left(\int_{0}^{1} r^{n-1}\left[\Phi_{1}(v(r))+1\right] d r\right)^{\frac{1}{\gamma}}
\end{aligned}
$$


where $K_{1}, K_{2}, K_{3}$ are constants independent of $u$. Finally, replacing (2.6) in (2.20) we obtain the second inequality in (2.11). This proves the lemma.

Lemma 2.4. Let $\hat{d}$ be as in Lemma [2.3, For each

$$
\epsilon \in\left(0, \frac{(n-1)(n+2-p(n-2))}{n(p-1)}\right)
$$

there exists a positive integer $k_{1}$ such that if $u$ is a radial solution of (1.1) with $|u(0)| \geq \hat{d}$ and $k \geq k_{1}$ interior nodal hypersurfaces, then

$$
J(u) \geq k^{\gamma+\epsilon} .
$$

Proof. By Lemma 2.3 there exists a positive integer $k_{0}$ such that if $u$ is a radial solution to (1.1) with $k \geq k_{0}$ interior nodal hypersurfaces, then $\left|v^{\prime}(1)\right| \geq 1+$ $2 \min \left\{\Phi_{1}(t) ; t \in \mathbf{R}\right\} \equiv K$ when $|v(0)| \geq \hat{d}$. We let $0=r_{0}$ and $0<r_{1}<\cdots<$ $r_{k+1}=1$ denote the zeros of such a solution $v$. Hence, by (2.3), we see that

$$
\left|v^{\prime}\left(r_{i}\right)\right| \geq K \quad \text { for } \quad i=1, \ldots, k .
$$

Let $\sigma:=(1-p) /((n+2)-p(n-2))<0$. By (2.21)

$$
(\gamma+\epsilon) \sigma-\sigma+1>0 .
$$

For each $i=1,2, \ldots, k$, let us define $v_{i}(r):=v\left(r_{i} r\right), r \in[0,1]$. An elementary calculation shows that $v_{i}$ satisfies

$$
\left\{\begin{aligned}
v_{i}^{\prime \prime}(r)+\frac{n-1}{r} v_{i}^{\prime}(r)+r_{i}^{2} f\left(v_{i}(r)\right) & =0, \quad r \in(0,1], \\
v_{i}^{\prime}(0)=v_{i}(1) & =0 .
\end{aligned}\right.
$$

Since $v_{i}$ has $i-1$ zeros in $(0,1)$, by Theorem 2 and Hölder's inequality, we see that

$$
\begin{aligned}
i & \leq c_{1} r_{i}^{n} \int_{0}^{1} r^{n-1}\left|f^{\prime}\left(v_{i}(r)\right)\right|^{\frac{n}{2}} d r \\
& \leq c_{2} r_{i}^{n} \int_{0}^{1} r^{n-1}\left[\Phi_{1}\left(v_{i}(r)\right)+1\right]^{\frac{1}{\gamma}} d r \\
& \leq c_{3} r_{i}^{n}\left(\int_{0}^{1} r^{n-1}\left[\Phi_{1}\left(v_{i}(r)\right)+1\right] d r\right)^{\frac{1}{\gamma}},
\end{aligned}
$$

where $c_{1}, c_{2}, c_{3}>0$ are constants independent of $v$. Now, by the definition of $v_{i}$ and Pohozaev's identity (see (2.6) ), we get

$$
\left|v^{\prime}\left(r_{i}\right)\right|^{2}=\int_{0}^{1} r^{n-1} \Phi_{1}\left(v_{i}(r)\right) d r .
$$

Combining (2.23), (2.25), and (2.26) we infer that

$$
C i \leq r_{i}^{n}\left|v^{\prime}\left(r_{i}\right)\right|^{2 / \gamma}
$$

here $C>0$ is a constant independent of $v$. Pohozaev's identity (see (2.6) $)$ implies that $r_{i}^{n}\left|v^{\prime}\left(r_{i}\right)\right|^{2} \leq\left|v^{\prime}(1)\right|^{2}+K \leq\left|v^{\prime}(1)\right|^{2} / 2$. This together with (2.27) shows that

$$
C i\left|v^{\prime}\left(r_{i}\right)\right|^{2(1-1 / \gamma)} \leq\left|v^{\prime}(1)\right|^{2} .
$$

From Lemma 2.1 and Lemma 2.3 we see that for $k$ to be sufficiently large $C_{1}\left|v^{\prime}(1)\right|^{2}$ $\geq 2 \bar{C}_{1}$. Suppose now that $J(u)<k^{\gamma+\epsilon}$. Since $1-p=2 \sigma(p+1)(1-1 / \gamma)$, (2.28) implies that

$$
\left|v^{\prime}\left(r_{i}\right)\right|^{\frac{1-p}{p+1}}>\left(\frac{2}{C C_{1}}\right)^{\sigma} k^{(\gamma+\epsilon) \sigma} i^{-\sigma} .
$$


Thus by Lemma 2.2 we have

$$
\begin{aligned}
1=\sum_{i=0}^{k}\left(r_{i+1}-r_{i}\right) & \geq C_{2} \sum_{i=1}^{k}\left|v^{\prime}\left(r_{i}\right)\right|^{\frac{1-p}{p+1}} \\
& >C_{2}\left(\frac{2}{C C_{1}}\right)^{\sigma} k^{(\gamma+\epsilon) \sigma} \sum_{i=1}^{k} i^{-\sigma} \\
& \geq\left(\frac{2}{C C_{1}}\right)^{\sigma}\left(\frac{C_{2}}{1-\sigma}\right) k^{(\gamma+\epsilon) \sigma-\sigma+1}
\end{aligned}
$$

which is a contradiction for large $k$ due to (2.24). This proves the existence of $k_{1}$, and the lemma is proved.

\section{Proof of Theorem 1}

Let $\left\{u_{i}\right\}$ be as in (1.8). By (1.9),$\delta \gamma<(n-1)(n+2-p(n-2)) /(n(p-1))$. Let $\epsilon$ be such that $\delta \gamma<\epsilon<(n-1)(n+2-p(n-2)) /(n(p-1))$, and $k_{1}$ as in Lemma 2.4 .

Since $J\left(u_{i}\right) \rightarrow+\infty$ as $i \rightarrow \infty$, we may assume that $\left|u_{i}(0)\right|>\hat{d}$ if $u_{i}$ is radial. Hence, since the number of interior nodal hypersurfaces tends to infinity as $|u(0)|$ tends to infinity (see (1.13) in [7]), we may also assume that if $u_{i}$ is a radial solution, then it has at least $k_{1}$ interior nodal hypersurfaces. Let $T>k_{1}^{\gamma+\epsilon}$. By (1.8), equation (1.1) has at least $\left(T / M_{1}\right)^{1 / \alpha}-1$ solutions with $J\left(u_{i}\right) \leq T$. On the other hand, by Lemma 2.4 if $u$ is a radial solution to (1.1) and $J(u) \leq T$, then $u$ has at most $T^{1 /(\gamma+\epsilon)}+1$ interior nodal hypersurfaces. Since, by hypothesis, there are at most $M_{2} k^{\delta}$ radial solutions with $k \geq k_{1}$ nodal interior hypersurfaces, equation (1.1) has at most $M_{2}\left(T^{1 /(\gamma+\epsilon)}+2\right)^{1+\delta}+1$ radial solutions $u$ with $J(u) \leq T$. Thus there are at least

$$
\left(T / M_{1}\right)^{1 / \alpha}-M_{2}\left(T^{1 /(\gamma+\epsilon)}+2\right)^{1+\delta}-2
$$

nonradial solutions to (1.1). Since $\alpha \leq \gamma$ and $\delta \in(0, \epsilon / \gamma)$, the quantity in (3.1) tends to infinity as $T$ tends to infinity. This proves Theorem 1 .

\section{Proof of Theorem 3}

For $d \in \mathbf{R}$ let $y(t, d)$ be the solution to the initial value problem

$$
\left\{\begin{aligned}
y^{\prime \prime}(r)+\frac{n-1}{r} y^{\prime}(r)+|y(r)+a|^{p-1}(y(r)+a) & =0, r \in(0,1], \\
y(0)=d, \quad y^{\prime}(0) & =0 .
\end{aligned}\right.
$$

Let $w(t, d)=y(t, d)+a$. Clearly $w$ satisfies the initial value problem $w^{\prime \prime}(r)+$ $\frac{n-1}{r} w^{\prime}(r)+|w(r)|^{p-1} w(r)=0$ in $[0,1], w(0)=d+a, w^{\prime}(0)=0$. Since $p \in$ $(1,(n+2) /(n-2))$, by Lemma 2.2 of [7], there exists $d_{1}>0$ such that if $|d| \geq d_{1}$, then

$$
\left(w_{r}(1, d)\right)^{2}+2|w(1, d)|^{p+1} /(p+1) \geq 1+2|a|^{p+1} /(p+1) .
$$

Because of (2.3), if $y(1, d)=0$ and $y(r, d)=0$ for some $r \in(0,1)$, then $\left|y_{r}(r, d)\right|>0$.

If $a \leq 0$, then $|t+a|^{p-1}(t+a)$ is bounded from above on $\left[0, d_{1}\right]$. Similarly, if $a>0$, it is bounded from above on $\left[-d_{1}, 0\right]$. Let $K$ be a corresponding upper bound. 
Assuming $a \leq 0$, by the Sturm comparison theorem, between two consecutive zeros of the solution to $z^{\prime \prime}+((n-1) / r) z^{\prime}+K z=0, z^{\prime}(0)=0, z(0)=1$, there can be at most two zeros $y(\cdot, d)$. Therefore $v$ cannot have more than $k_{1}=2 k_{0}+2$ zeros in $(0,1)$, where $k_{0}$ is the number of zeros of $z$ in $(0,1)$. Similarly for $a \geq 0$.

An elementary calculation shows that $W(r, d)=(2 /(p-1)) w(r, d)+r w_{r}(r, d)$ satisfies

$$
\left\{\begin{aligned}
W^{\prime \prime}(r)+\frac{n-1}{r} W^{\prime}(r)+p|w(r)|^{p-1} W(r) & =0, \quad r \in(0,1], \\
W(0)=(2 /(p-1))(d+a), \quad W^{\prime}(0) & =0 .
\end{aligned}\right.
$$

Since $w_{d}(r, d)$ also satisfies the second order linear equation in (4.3) and $w_{d}(0, d)=1$ we have $w_{d}(r, d)=((p-1) /(2(d+a))) W(r, d)$. Since $y(r, d)=0$ when $w(r, d)=a$, by the definition of $W$ and (4.2), we see that

$$
y_{d}(r, d) y_{r}(r, d)=w_{r}(r, d)\left((2 / p-1) a+r w_{r}(r, d)\right)>0 .
$$

Thus, by the implicit function theorem (see also [12]), the zeros of $y(r, d)$ are a decreasing function of $d$, for $d \geq \hat{d}$. In particular for each $k$ there are at most two values of $d$ for which $y(\cdot, d)$ is a solution to (2.1) with exactly $k$ zeros in $(0,1)$. Thus there can be at most two solutions to (1.1) with $k>k_{1}$ interior nodal hypersurfaces. Since $p \in(1,(n+2) / n)$, (1.8) follows from the results of [1. Combining this with the definition of $k_{1}$, by Theorem 1 , Theorem 3 follows.

\section{ACKNOWLEDGEMENTS}

The authors wish to thank the referee for helpful comments.

\section{REFERENCES}

1. A. Bahri and P.-L. Lions, Morse index of some min-max critical points. I. Application to multiplicity results, Comm. Pure Appl. Math. 41 (1988), 1027-1037. MR 90b:58035

2. H. Berestycki and P.-L. Lions, Nonlinear scalar field equations. II. Existence of infinitely many solutions, Arch. Rat. Mech. Anal. 82 (1983), 347-375. MR 84h:35054b

3. T. Bartsch and M. Willem, Infinitely many nonradial solutions of a Euclidean scalar field equation, J. Funct. Anal. 117 (1993), 447-460. MR 94k:35093

4. A. Castro, J. Cossio, and J. M. Neuberger, On multiple solutions of a nonlinear Dirichlet problem, Nonlinear Analysis TMA 30 (1997), 3657-3662. MR 98h:35070

5. A. Castro and M. Finan, Existence of many sign-changing solutions to a superlinear Dirichlet problem on thin annuli, Topological Methods in Nonlinear Analysis. 13 (1999), 273-280. MR 2000j:35092

6. A. Castro and M. Finan, Existence of many positive nonradial solutions for a superlinear Dirichlet problem on thin annuli, Nonlinear Differential Equations, Electron. J. Diff. Eqns., Conf. 05 (2000), 21-31. MR 2001i:35116]

7. A. Castro and A. Kurepa, Infinitely many radially symmetric solutions to a superlinear dirichlet problem in a ball, Proc. Amer. Math. Soc. 101 (1987), 57-64. MR 88j:35058

8. M. Cwikel, Weak type estimates for singular values and the number of bound states of Schrödinger operators, Ann. Math. 106 (1977), 93-100. MR 57:13242

9. D. Fortunato and E. Jannelli, Infinitely many solutions for some nonlinear elliptic problems in symmetrical domains, Proc. Roy. Soc. Edinburgh Sect. A 105 (1987), 205-213. MR 88m:35057

10. W.-Y. Ding, On a conformally invariant equation on $\mathbf{R}^{n}$, Comm. Math. Phys. 107 (1986), 331-335. MR 87m:35066 
11. M. Struwe, Superlinear elliptic boundary value problems with rotational symmetry, Arch. Math. 39 (1982), 233-240. MR 84a:35097

12. E. Yanagida, Structure of radial solutions to $\Delta u+K(|x|)|u|^{p-1} u=0$ in $\mathbf{R}^{n}$, SIAM J. Math. Anal. 27 (1996), 997-1014. MR 98c:35047

Departamento de Matemáticas, Universidad de Córdoba, Montería, Colombia

E-mail address: haduen@hotmail.com

Division of Mathematics and Statistics, The University of Texas at San Antonio, San Antonio, Texas 78249

E-mail address: acastro@utsa.edu 